\title{
Intervención Social y SENAME: \\ Análisis crítico de discursos de mujeres interventoras
}

\author{
Social Intervention and Chilean child welfare system: \\ critical discourse analysis of women interventionist
}

Beatriz Toro Castillo ${ }^{1}$

\begin{abstract}
Resumen
El siguiente artículo tiene la finalidad de interpretar la práctica y significación interventiva de un grupo de mujeres interventoras del área de protección de SENAME, desde un enfoque epistemológico crítico. Metodológicamente, se realiza un análisis categorial de los hilos discursivos producidos por un grupo de mujeres que intervienen en este escenario, sumado al análisis de documentos, que en este caso corresponden a las bases técnicas de los programas en los cuales ellas trabajan, desde el enfoque propuesto por Jäger. El marco teórico que provee al análisis categorial, surge de las aportaciones de la teoría de la intervención social crítica de Karen Healy, Teresa Matus y Alfredo Carballeda. Las categorías teóricas se sitúan comprensivamente desde la teoría de la perfomatividad planteada por Butler y Austin, referencia que en este artículo se hace cuidadosamente. Por último, se dan a conocer algunas categorías teóricas emergentes, que nacieron al alero de la interpretación, en este caso, corresponden a elementos de la teoría freudiana y la teoría del desarrollo éticomoral de Gilligan.
\end{abstract}

Palabras Clave: SENAME, mujeres interventoras, intervención social clásica, intervención social crítica, performatividad.

\begin{abstract}
The following article has the purpose of interpreting the practice and interventional significance of a group of interveners' women in the SENAME's protection area from a critical epistemological approach. Methodologically, a categorical analysis of the discursive areas produced by a group of women involved in this scenario, at the same time with the analysis of documents, is carried out, which in this case correspond to the technical bases of the programs they work, from the focus proposed by Jäger. The theoretical framework provides the categorical analysis, arises from the contributions of the critical-social intervention theory of Karen Healy, Teresa Matus and Alfredo Carballeda. On the other hand, the theoretical categories are comprehensively situated from the perfomativity theory proposed by Butler and Austin. Finally, emerging theoretical categories are showed, performing the interpretation; correspond to elements of the Freudianan theory and the ethical-moral development of Gilligan theory.
\end{abstract}

Key words: SENAME, interveners' women, classic -social intervention, critical-social intervention, performativity. 


\section{Introducción}

El Servicio Nacional de Menores (SENAME), es una institución perteneciente al Ministerio de Justicia y Derechos Humanos de Chile, que tiene como objetivo central prevenir, promover, proteger y restituir los derechos vulnerados de niñas, niños y adolescentes (SENAME, 2020). Para cumplir con ello, genera una oferta programática sujeta a tres áreas interventivas: Responsabilidad Penal Adolescente (RPA); Adopción; y Protección (SENAME, 2020). El ejercicio de estas áreas es realizado por centros de administración directa de la institución, y por organismos colaboradores (conocidos como OCAs).

Este estudio se focaliza en la programación del área de Protección de SENAME, desarrollada por las OCAs. El marco interventivo de estas instituciones, está encuadrado bajo dos documentos, las orientaciones técnicas, que brinda SENAME, y su proyecto de licitación. Ambos documentos presentan modelos de intervención declarados, tales como enfoque de derecho, enfoque ecológico, ampliación sociocultural, enfoque sistémico, activación de redes, entre otros (Contreras, Rojas, Contreras, 2015). Además, los proyectos de licitación cuentan con protocolos de ingreso, frecuencia, flujo de atención, e instrumentos específicos para la evaluación (Schöngut-Grollmus, 2017).

La aplicabilidad de esta estructura interventiva institucionalizada, aparentemente difiere de la subjetivación de las y los interventores, ya que, por ejemplo, en lo que respecta a los modelos de intervención, parece que se reduce exclusivamente al concepto de competencia parental, siendo utilizada desde los juicios que sostienen las y los profesionales respecto a lo que creen que los niños, niñas y adolescentes (NNA) y sus familias carecen. Esto conlleva a que, a pesar de la existencia de objetivos de intervención más amplios, se termina interviniendo sobre objetivos focalizados (Contreras, Rojas, Contreras, 2015). A su vez, Schöngut-Grollmus (2017) señalan que, a partir de la reducción teórica, disminuye la posibilidad de usar otros marcos de acción, privilegiando responder a las metas institucionales y descuidando necesidades sentidas por los NNA, debido a que el enfoque y/o instrumento no logra identificarlas.

Ante ambos antecedentes, se interpreta que la declaración interventiva de la institución, se encuentra validada en el paradigma clásico de la intervención social (Healy, 2001).

Al revisar la Revista académica de la Unidad de Investigación de SENAME, se puede encontrar una amplia gama de artículos que aluden a un paradigma cartesiano-postivista. Por ejemplo, se hace referencia a estrategias de intervención como la "visita supervisada" la que es comprendida como una instancia segura para la interacción entre NNA y sus familias que no poseen los cuidados personales, siendo las o los interventores quienes legitiman el espacio seguro (Molina y Moraga, 2019). O la aseguración de un proceso interventivo de calidad, sujeto a competencias que realzan el conocimiento técnico y profesionalista, tales como las competencias metodológicas, personales, participativas, y técnicas, sin hacer referencia la integración de los saberes de quienes son intervenidas/os (Aguilera, 2018).

Considerando estos aspectos, el presente artículo tiene la intención de analizar el marco teórico-técnico de algunos programas de intervención de SENAME, a partir de la aproximación a un enfoque clásico o crítico de la intervención social, acudiendo a constructos, tales como perfomatividad, intervención social crítica, e intervención social clásica, con tal de facilitar el análisis tanto de las orientaciones técnicas como del discurso de quien interviene.

Por lo tanto, el objetivo que pretende alcanzar este artículo es interpretar los hilos discursivos que generan mujeres interventoras del área de Protección de SENAME y las orientaciones técnicas de estas, en relación al constructo de intervención social. 


\section{Intervención Social}

La Intervención Social (I.S.) es un dispositivo discursivo (Carballeda, 2008 a) y simbólico (Matus, 1999), constructor de subjetividades y sistemas comprensivos que se circunscriben en torno a un orden normativo contextualizado territorial e históricamente- que define el objeto sobre el cual se actuará, con el objetivo de disciplinarlo y controlarlo (Carballeda, 2008 b; Matus, 1999).

Para la consolidación de este poder controlador y disciplinador, la I.S. debe instalarse en la cotidianidad de la población, para construir de este modo, parte de su realidad (Egas y Salao, 2011), por medio de la acción/actuación de alguna profesional (actriz), sobre aquello que se interprete como un problema social complejo (Saavedra, 2015; Carballeda, 2008 a). A partir de esto es que se asume que, toda acción inteventiva involucra, inalienablemente, un acto interpretativo (Matus, 2006).

A partir de la aproximación hecha al constructo, se extraen aristas conceptuales: a) actrices/actores; b) problema social complejo; c) actuación/acción; d) escenario de la I.S; y e) sistema normativo, las que se desarrollarán a continuación con el propósito de profundizar en los constructos.

\section{Actuación/Acción de la I.S.}

La Intervención Social se evidencia como tal, gracias a la existencia de un fluido de actuaciones/acciones que la encarnan, por medio de una corporeidad epistémica (Saavedra, 2015).

La actuación/acción de la I.S., por ende, la corporeidad epistémica, es el resultado del proceso que sitúa al conocimiento teórico-epistemológico, como "el pensamiento que produce un impulso práctico" interventivo (Matus, 2006, p. 61). Este proceso va transformando la realidad, ya que va entregando nombres/categorías (Matus, 2006) que encierran valores, creencias, actitudes, contextos políticos y filosóficos (Saavedra, 2015) específicos para la I.S.; por lo tanto, conocer y estar consciente del enfoque teórico-epistemológico que se encuentra a la base del desenvolvimiento de actuación/acción interventiva, sirve para comprender y desarrollar esta misma (Healy, 2001). A partir de esto es que la relación establecida entre actuación/acción y teoría, está dada porque esta última otorga significado a la primera.

Toda esta operatividad no es azarosa ni inocua, sino más bien, posee un telos determinado; en este caso, en lo que concierne a la I.S., el telos se dirige a otorgar integración y bienestar, tanto personal como comunitario, por medio de estrategias que, si bien se buscan instalar en la cotidianidad de la población, se realizan artificiosamente en estas mismas (Saavedra, 2015).

Por otra parte, lo que se signifique como bienestar e integración, estará supeditado a lo que la ideología dominante signifique como tal, por lo que, toda actuación realizada en este escenario es cómplice de la reproducción de contextos y estados opresores (Healy, 2001).

En síntesis, existe un tejido relacional entre actuación/acción, enfoque teórico-epistemológico e ideología dominante que deja a la primera como el producto y expresividad corpórea de la segunda, la cual a su vez está supeditada y autorizada por la ideología dominante que deposita su telos en esta, y que logra expresarse por medio de la primera.

\section{Actoras/es de la intervención social}

La gama amplia y compleja de acciones/actuaciones se concreta en una configuración ontológica.

Esta configuración ontológica de la intervención social, es corporeizada por actrices y actores a los que se les asigna una performance, construida históricamente, y que traslapa un sentido de pertenencia identitario (Carballeda, 2008 b), que posiciona en la escena de I.S. (Matus, 1999 en Saavedra, 2015), no de forma creativa, sino situada y subjetiva del papel a interpretar. 
A partir de esto, se identifican, como identidad, tres actrices/actores claves: la institucionalidad, interventora/ or e intervenida/o.

El cómo se signifique cada una/o de las/os actrices/actores de la intervención, dependerá de la perspectiva/ paradigma teórico al cual uno adscriba. En este artículo se hace la lectura desde dos líneas teóricas, la intervención social clásica, y la intervención social crítica.

Desde la intervención social clásica, la y el interventora/or es concebida como una sujeta/o experta/o, cuyo objetivo es controlar/usar y disciplinar (Carballeda, 2008 b). La y el interventora/or, según lectura de Dominelli y MacLeod, se significa como auxiliadora dentro de los escenarios y objetivos del proceso de la intervención social (En Healy, 2001) que, gracias a las capacidades superiores que posee, permiten cuidar y salvar a una población que está en constante riesgo (Matus, 2006).

Mientras que, desde la teoría de la I.S. crítica, la/el intervenida/o, es significada/o como excluida/o social (Saavedra, 2015), que contiene el malestar social a intervenir (Moreno, 2013). Esta característica performática significa a las/ os sujetas/os de intervención, como las/os sujetas/os devaludas/os, "des-afiladas, des-calificadas, de-lincuentes, des-capacitado y des-poseídos" (Matus, 2006, p.6.).

Por otra parte, las/os interventoras/es son significadas/os como privilegiadas/os, ya que contienen poder, que les permite movilizar voluntades y configurar cuerpos. El discurso de las/os interventoras/res norma las prácticas de las/os intervenidas/os (Saavedra, 2015). A partir de esto, es que, desde la teoría crítica, se considera como relevante que la/el interventora/or desarrolle procesos de autoconscientización, que involucre una práctica reflexiva y crítica, con tal de orientar la intervención lejos del ejercicio de dominación y opresión; este desarrollo de consciencia debe estar entretejido con el despliegue de un trabajo comprometido ético y técnico (Healy, 2001).

Quizás, la relación más directa y cercana dentro del contexto de I.S. sea aquella que se establece entre la/ intervenida/o e interventora/or. Y para comprenderla como tal, también depende de la línea teórica desde la cual se aproxime.

Desde la teoría clásica de la I.S., primero, se enfatiza una relación asimétrica y desigual entre interventora/ or e intervenida/o; segundo, no pretende establecer una relación transparente entre ambas/os (Healy, 2001); tercero, establece relación binaria entre conocimiento vs. saber, donde las/os interventoras/es se posicionan desde el conocimiento (cientificista-experto), y las/os intervenidas/os desde el saber, teniendo supremacía y valoración el primer sistema; ante esto, el conocimiento/saber que puede llegar a expresar la población, está lo suficientemente menospreciado por la comunidad experta, al ser significada como un conocimiento que asegura el fracaso (Matus, 2006); y cuarto, la dinámica generada entre ambas/os actrices/actores, genera binarismo de dominadora/or (interventora/or)-dominada/o (intervenida/o).

Por otra parte, en lo que respecta a la teoría crítica de la I.S., primero, reconoce y visualiza la tradición relacional clásica existente entre interventora/or e intervenida/o tejida en el escenario de la intervención social; segundo, a partir del primer punto, visibiliza y concientiza respecto a la relación de poder existente entre estas dos actrices/actores de la intervención, por lo tanto, busca promover relaciones lo más equitativas entre estas/o; tercero, desprendiéndose del punto anterior, la teoría crítica promueve la identificación entre interventora/or e intervenida/o, haciendo saliente identidades y experiencias en común; cuarto, en lo que respecta al conocimiento/saber, significa los saberes y conocimiento de la población a intervenir, como significativos y relevantes para el proceso de intervención, situándolos en un escenario equitativo en lo que respecta al conocimiento cientificista, es decir, se considera necesario dar posición significativa al conocimiento entregado por la/el intervenida/o, y no dejarlo subyugado y devaluado en función del conocimiento científico (Healy, 2001). 
El punto crítico que presenta las claves de la teoría crítica, es que raya en la posibilidad de situar a la/el intervenida/o, como alguien totalmente desempoderada/o, incapaz de generar dinámicas de coerción, mientras que, por otro lado, sitúa a las/los interventores/es como la autoridad por excelencia. Al reconocer, desde la teoría crítica, la asimetría de poder, se abre la posibilidad de establecer como objetivo el minimizar dicha dinámica, mas, corriendo el riesgo de caer en una generalización exagerada de la posición de la/el interventor/a como privilegiada/o, y la posición de la /el intervenida/o, como desventajosa (Healy, 2001).

\section{Problema Social de la I.S.}

La I.S. puede ser comprendida como una disciplina, que, dentro de sus objetivos, busca potenciar la integración social, con tal de asegurar la estabilidad de las pautas, estructuras y roles normativos (Matus, 1999). Para concretar dicho objetivo, la disciplina crea e identifica interpretativamente, escenarios y actrices/actores sociales, y los significa como problemas sociales complejos.

La relación de estos tres elementos, escenario, actrices/actores y problemas, se basa en laidea de disfuncionalidad, desviación y deseable/no deseable, según lo defina el marco normativo, pero, es ante lo no deseable que la intervención social opera, con la finalidad de ir normalizando aquellos discursos transgresores, para disciplinar (Saavedra, 2015).

Por otra parte, los problemas sociales complejos no son rígidos ni estáticos, por el contrario, se caracterizan por su movilidad y constantes cambios, lo que da paso a la creación de nuevos guiones, actuaciones, actrices/ actores y acciones, sobre los cuales, el grupo de profesionales, con ayuda de los dispositivos de intervención, intervienen sobre el padecimiento de la población objeto (Carballeda, 2008 a).

De todas maneras, independiente del dinamismo de los problemas sociales complejos, siempre a la base de estos se encontrará una población significada patológicamente. Esta condición escénica de la I.S., habla de patologías sociales, por lo que todo aquel/aquella que se sitúe desde esta significación, se categoriza, desde la lógica de la normatividad hegemónica, como "des-afiladas, des-calificadas, de-lincuentes, des-capacitado y des-poseídos" (Matus, 2006, p.6.).

Esta aproximación de la I.S. y su relación con los problemas sociales complejos, surge a partir del juego establecido entre Norma e I.S. Este juego se sustenta en el temor a la transgresión de la primera, lo que da paso a la necesidad de eliminar y controlar la diferencia, para evitar el caos y desorden, al poner en riesgo la Norma-, debiendo ser minimizado o extinguido (Matus, 2006; 1999). ¿De qué manera se extingue? por medio del castigo (Matus, 2006). El mejor castigo identificado/interpretado en la investigación, es la exclusión. Esto involucra y se asocia con la desigualdad social y procesos de estigmatización (Carballeda, 2008), los cuales generan espacios de segregación social (Fantova, 2007).

El problema de esta lógica, o modo de funcionamiento de la I.S., es que genera una manera reducida de aproximación hacia las sujetas sobre las cuales se intervienen. Si bien, ya se tiene claro que la I.S. trabaja sobre diversas y determinadas formas de exclusión, es respecto a la categoría identitaria que se filtra y se define la forma de la exclusión (Matus, 2006), la identidad nombra, identifica y posiciona, por lo tanto, limita, significa y ubica a la/el sujeta/o que es nombrado, dentro de los márgenes de la categoría con la que se le interpeló.

\section{Escenario de la I.S.}

Tanto Butler como Carballeda metaforiza la vida como una obra teatral, donde cada espacio, contexto y/o dimensión, es una escena, en la cual actrices/actores desempeñan un rol/papel. La I.S. no es la excepción. Carballeda (2008 b), asume sin mayor rodeo, que la I.S., es un escenario con actrices/actores, un guion/texto, y una trama.

Estructuralmente, la escena de la I.S. se constituye de historia, contexto e ideología, expresados en las políticas sociales y la cultura, que delimitan el cuerpo de la escena y trama de la I.S. (Carballeda, 2008 b). 
Dentro de la estructura escénica, también dinamizan elementos materiales y corpóreos, tales como las/os actrices/actores y el territorio. Entre estos elementos se genera un diálogo que define circunstancias, problemas, demandas, mandatos y papeles (Carballeda, 2008 a).

\section{Sistema Normativo / Orden Simbólico de la Intervención Social}

Tal como se menciona en párrafos anteriores, la I.S. actúa sobre lo que se considere como un problema social. El punto en esto es, ¿Qué hace que se considere, o más bien, se signifique, como un problema social? Es la interpretación de un escenario sujetado hacia un orden simbólico que encuadra la complejidad de significantes conducente a dicha interpretación.

El orden simbólico, el cual se establece como sistema normativo, provee sentido e historia (Matus, 2006). Esta simbolización no llega a ser tan solo una referenciación abstracta de la realidad, sino más bien, como había mencionado anteriormente, presenta un correlato material, como palabras, imágenes, performances, y ritos, los cuales contienen la semiótica del símbolo, por medio de la relación entre signo y significante (Ortega, 2015).

Por otra parte, como una de las tantas formas de expresar significado, lo simbólico genera orden y prácticas (Ortega, 2015), y que, en el caso de la intervención social, se traduce en una condición escénica específica, que habla de patologías sociales, faltas y carencias (Matus, 2006).

Bajo este concepto, las/os interventoras/es juegan un rol interesante. Primero, el orden simbólico / sistema normativo, permite que las/os interventoras/es, normen las prácticas de las/os intervenidas/os, ya que su discurso posee el poder de movilizar voluntades y configurar cuerpos (Saavedra, 2015). Segundo, la relación entre norma e interventora no es unilateral, es decir, la norma no coapta por completo los discursos de las interventoras, y esto es gracias a que estas poseen historia, valores, representaciones, y creencias previas a la intervención social, y que merman la visión que poseen del otro, por tanto, también reducen cómo construyen el proceso de I.S. (Carballeda, 2008 a). Esto se significa como un punto de fuga y subversión en lo que respecta al orden simbólico de la intervención social

\section{Metodología}

Los elementos metodológicos que estructuraron la investigación fueron: a) metodología cualitativa; b) análisis de datos bajo la lógica de análisis de discurso propuesta por Jäger (2003); c) la población consistió en cinco mujeres profesionales del área de protección de SENAME, específicamente dos trabajadoras sociales y tres psicólogas, por lo que de ahora en adelante se hablará solo de interventoras; d) la técnica de producción de datos fue análisis de documento y entrevista semi-estructurada.

En relación a la metodología cualitativa utilizada en este estudio, es entendida como aquella que valida las relaciones sociales situadas, locales y temporales, junto con validar la subjetividad de quien investiga para construir el conocimiento, integrando, y no anulando, divergentes y heterogéneas perspectivas y enfoques para aproximarse al objeto de estudio, construyéndolo e interpretándolo de manera compleja, ya que, distintas formas de ver, conciben diversas formas de ser, sin que esas diversas formas, sean consideradas contradictorias y falsas (Flick, 2004)

El escenario donde dinamizan las relaciones sociales -como objeto de estudio- y la reflexión de quien investiga, se enmarcan dentro de la cotidianidad misma de esta relación, más bien, se enmarca dentro de la espontaneidad de la interacción, a diferencia de la perspectiva positivista, donde cada elemento de este escenario se construye de manera artificial, para así obtener un conocimiento correcto (Fick, 2004). Esto quiere decir que, el realismo ingenuo, que sustenta a la investigación cuantitativa - así como también, más masificado-, deja de ser relevante para la investigación cualitativa, pues su foco no es llegar a un conocimiento objetivo, sino más bien, su foco está puesto en poder resaltar y validar un conocimiento sustentado en subjetividades y cotidianidades (Álvarez y Juergenson, 2003). 
Otro elemento relevante, del enfoque de la investigación cualitativa de este estudio, es el cómo concibe la relación de investigada e investigadora. Las visibiliza como dos entes que concluyen y co-construyen el conocimiento (Flick, 2004). Esto involucra reconocer, primero, que es imposible separar a la investigadora del proceso de intervención, así como también es imposible separarla de toda su performance de mundo, y segundo, es imposible que ambas no se influyan mutuamente (Álvarez y Juergenson, 2003).

En relación al diseño de investigación, se utilizó el modelo diamante o rombo. Este diseño implica el siguiente proceso, primero, definir claramente un problema, luego interiorizarse primeramente en la teoría, y a partir de allí construir las categorías analíticas a priori y posteriori, y finalmente analizar y sintetizar los datos analizados (Bivort y Martínez, 2012).

En relación al método de investigación, se utilizó el análisis crítico de discurso propuesto por Jäger (2003). Epistemológicamente, en relación al conocimiento, este método refiere que surge de forma histórica y contextualizada, en un lugar y tiempo determinado, dando como resultado discursos que fluyen, modelando individualidades y sociedad por medio del ejercicio del poder (Jäger, 2003). En relación a la postura de quien investiga, se asume que no se encuentra al margen del discurso que va analizando, por lo tanto, estarán presente sus valores y normas.

En relación al objeto de estudio, es el discurso, el cual, para el autor es significado como un conocimiento societal que se acumula histórica, experiencial y entretejidamente. Lo que busca este método, es destejer dicho entramado e interpretar los símbolos presentes con tal de conocer la representación de la realidad de quien produce el discurso. Pero la característica que más revela el discurso, y que es una consideración central de esta investigación, es que encierra poder y es supraindividual (Jäger, 2003)

En lo que respecta a la operatividad del análisis crítico discursivo, se trabajó en dos niveles, primero el conceptual, y segundo, dependiente del primero, la diagramación procesual de la recogida y análisis de datos.

A nivel conceptual, se usó el concepto de hilo discursivo y fragmento discursivo propuesto por Jäger (2003).

En lo que respecta al hilo discursivo, corresponde a la uniformidad temática presente en el discurso. Este posee la cualidad de ser diacrónico y sincrónico; esto tiene la finalidad de identificar lo que es dicho, lo que fue dicho y lo que será decible (Jäger, 2003).

En lo que respecta a los fragmentos discursivo, vendría a reemplazar lo que comprenden los hilos discursivos, es decir, el texto (Jäger, 2003). Pero la idea de fragmento es más reducida que la idea de texto, pues este último, puede llegar a convocar una variedad temática, mientras que el fragmento, solo hace referencia a un tema puntual (Jäger, 2003). En base a esto, se puede señalar que, el trabajo en relación a los fragmentos discursivos, está sujeto a profundizar los hilos discursivos, puesto que varios fragmentos confluyen para dar cuenta de un hilo discursivo (Jäger, 2003). A partir de la última frase, se desprende que la relación entre texto e hilo discursivo, es que varios hilos discursivos forman un texto, lo que permite deducir que varios fragmentos están contenidos en un texto (Jäger, 2003).

En lo que respecta a la diagramación procesual del análisis, se inició por medio de la recolección/producción de datos, a través de dos técnicas. Por una parte, la entrevista semiestructurada en profundidad, la que permite aproximarse al conjunto de significados que posee la entrevistada en relación a sus experiencias (Álvarez y Juergenson, 2003), percepciones e impresiones (Quivi y Van Carnpenhoudt, 2005). Estos significados son registrados por la entrevistadora para posteriormente ser interpretados por esta misma (Álvarez y Juergenson, 2003). Acarrea la necesidad de contextualizar a la persona entrevistada, realizando una breve descripción y justificación del motivo de investigación (Álvarez y Juergenson, 2003).

Se debe construir un guion donde se reflejan los temas deseados a tratar por la entrevistadora (Corbalette, 2007), el cual no es rígido, por lo que permite la expresión de preguntas contingentes relacionadas con la temática tratante de la investigación (Quivi y Van Carnpenhoudt, 2005). La profundidad de esta está en contener el principio de especificidad, es decir, se busca conocer significados específicos y no generales (Álvarez y Juergenson, 2003). 
En segundo lugar, se utilizó el análisis de documento, el cual se caracteriza por permitir conocer la ideología, sistema de valores, la cultura (Quivi y Van Carnpenhoudt, 2005) y sistema de significados, presentes en documentos institucionales, por medio de la interpelación de la investigadora (Corbalett, 2007). Permite analizar experiencias macrosociales, otorga información para interpretar el devenir histórico y cambios sociales, y analiza cambios en instituciones (Quivi y Van Carnpenhoudt, 2005). Existen dos tipos de análisis de documento, el dependiente de personas y dependiente de instituciones. En esta investigación se trabajó con documentos institucionales. Los documentos institucionales se caracterizan por ser producidos por instituciones o individuos en contexto institucionalizado, y que toman el carácter de poseer contenido público (Corbalett, 2007). El nivel de análisis de los documentos fue de tipo soterrado, es decir, se analiza su contenido y sus significados de manera parcelada, asumiendo que no necesariamente existe relación entre sí, por lo que se debe interpretar separadamente. En el fondo, el análisis puede llegar a abordar el texto en dos niveles de codificación e interpretación, en relación a sí mismo, como también, fuera de sí (Ruiz, 2003).

El análisis de documento, posee la ventaja de crearse independiente a la acción de la investigadora, por lo que no se ve interferida por aspectos relacionales -como en el caso de la entrevistada y entrevistadora(Corbalett, 2007), y, por otro lado, hay un alto grado de confiabilidad en relación a su contenido (Quivi y Van Carnpenhoudt, 2005).

Especificando el análisis de documento, estos fueron las orientaciones técnicas correspondientes a los programas en los cuales ejercían las profesionales entrevistadas, tales como Oficina de Protección de Derecho (O.P.D.), Programa de Intervención Especializda (P.I.E.), Programa de Familia de Acogida Especializada con Programa de Protección Especializada (F.A.E.-P.R.O.), Centro de Residencia Modalidad Residencia con programa de Protección Especializado (R.E.M. - P.E.R.) y Diagnóstico Ambulatorio (D.A.M.).

El segundo y último paso de esta diagramación procesual, es el análisis de los datos recolectados/producidos. La técnica empleada para esto fue la construcción de matriz de coherencia.

La construcción de la matriz, inició con la identificación de los conceptos críticos y claves de los objetivos de investigación, para luego ser definidos desde el marco teórico. Al concluir su definición se buscó operativizarlos por medio de la construcción del instrumento de investigación que fue la pauta de preguntas, donde cada pregunta hacía referencia a los conceptos críticos y claves.

La construcción de la matriz, inició con la identificación de los conceptos críticos y claves de los objetivos de investigación, para luego ser definidos desde el marco teórico. Al concluir su definición se buscó operativizarlos por medio de la construcción del instrumento de investigación que fue la pauta de preguntas, donde cada pregunta hacía referencia a los conceptos críticos y claves.

\section{Intervención social}

\section{Enfoque teórico}

\section{Acción/actuación}

Sujetos de

intervención 


\section{Género}
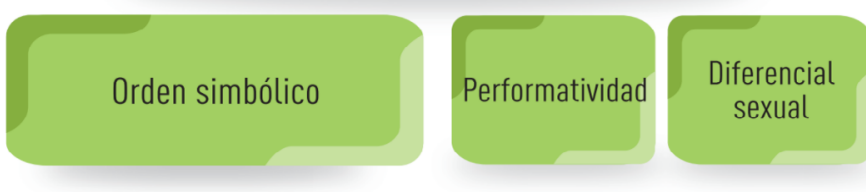

Relaciones

objetales

Construcción

Yoica

Gráfica 2. Matriz de coherencia. Concepto crítico: Género. Elaboración propia (2017)

Posterior a la construcción de los instrumentos, y desarrollo de las entrevistas e identificación de los documentos de análisis, se realizó el análisis interpretativo a nivel de contenido de cada uno de los textos, en relación a las categorías construidas y descritas en la matriz de coherencia.

Cabe mencionar que la entrevista en profundidad fue aplicada a cinco mujeres interventoras. Con cada una de ellas se sostuvo cinco encuentros, con una duración de dos horas promedio. Dos de las cinco entrevistas tenían como temática exclusiva la Intervención Social.

A partir del análisis de los datos sujeto a las categorías referidas en la matriz se interpretaron los fragmentos e hilos discursivos, creados desde la interpretación de quien investiga, y posteriormente fueron entretejidos con las categorías de análisis identificadas sobre intervención social presentes en la matriz de coherencia.

\section{Resultados}

Los resultados analizados bajo la metodología recientemente descrita, se dividen en dos áreas; primero, análisis de documentos pertenecientes a las orientaciones técnicas de cinco programas del área de protección de SENAME: OPD, REM-PER, PIE, FAE-PRO y DAM; segundo, se presenta el análisis de discurso construido por cinco profesionales mujeres interventoras, pertenecientes a cada uno de los programas recientemente mencionados.

Es relevante señalar que los resultados presentados a continuación se relacionan con una parte de la investigación, la que hace referencia a la intervención social. En lo que respecta a los resultados relacionados con la categoría género, solo se referirán aquellos que tributen al análisis del primero.

\section{Análisis de Orientaciones técnicas de programas de protección de SENAME}

El análisis de documento se realizó interpretando el texto (orientaciones técnicas) y creando los hilos discursivos por categoría arrojada en la matriz de coherencia.

\section{a) Enfoque Teoría}

Bajo esta categoría se interpretaron los siguientes hilos discursivos: sujeto moderno, ideal parental y metodología cartesiana-positivista.

Ante esto, la referencia está hecha, centralizadamente, en los aportes de Healy (2001). 
Los hilos discursivos interpretados apelan a una construcción clásica de la intervención social. Se interpreta de dicha manera porque (Healy, 2001): a) el sistema atributivo es de corte disposicional, al referir que la responsabilidad de cambio y problema corresponde a las familias que son intervenidas; b) las metodologías corresponden al paradigma moderno; c) no visualiza ni contempla la idea de estructura social como abordaje teórico para la aproximación interventiva; d) enfatiza una relación asimétrica entre la institución-interviniente y quien es intervenido; y e) no incorpora el concepto de poder, como constructo comprensivo del proceso de intervención.

Fantova (2007), refiere que la mirada clásica de la intervención social, busca satisfacer/gratificar, necesidades de la población en general, por medio de políticas públicas, con tal de mejorar su calidad de vida. Ante esto, primero, claramente la política pública es ejecutada por SENAME, y segundo, la necesidad a intervenir -se desprende-interpreta- corresponde a resguardar los derechos de las NNA, pero, aquello se realiza desde la dominación de sus familias, con tal de que estos se hagan cargo de aquello.

Ante esto, pareciera que, claramente, el enfoque teórico tomado por el área de protección de SENAME, habla sobre una mirada alejada por completo de fundamentos críticos de la intervención social, y reproduce discursos en torno a la mirada clásica (Healy, 2001).

Por otra parte, los antecedentes teóricos declarados por la institución, sumado a la interpretación realizada de estos, carecen de la reflexivilidad y conciencia ética que debiera poseer, según Carballeda (2008 a). Estas características no se encuentran plasmadas en ningún apartado de las orientaciones de los programas, cuestión preocupante sobre todo en lo que respecta al pronunciamiento ético, el cual marca los márgenes de aproximación/relación entre las sujetas/os de la intervención.

A esto se le suma la falta de una declaración epistemológica (Saavedra, 2015) coherente por parte de la institución, puesto que el análisis realizado de sus marcos pareciera la construcción mosaica, incoherente e irreflexiva de este.

Como punto aparte, y tomando el segundo hilo discursivo, se desprende como interpretación teórica, la relación entre teoría psicodinámica y el hilo ideal parental.

Ante esto se interpreta que, al igual que la teoría winniconiana, la cual plantea la existencia de una madre suficientemente buena (Winnicott, 1993; 1995), la cual se caracteriza por satisfacer favorable y amorosamente las necesidades del infante que cuida, pareciese ser, que, desde las pretensiones e interpretaciones de la institución, debiese existir una parentalidad suficientemente buena. Todo aquello que se escapa de dicho ideal, es patologizado, devaluado y destinado a la intervención.

\section{b) Actuación/acción de la intervención social}

En esta categoría se interpretaron los siguientes hilos discursivos: ciclo tecnológico de la intervención social, utilitarismo relacional, evaluación coercitiva, disposición legal, evidencia panóptica, abanico de acción proteccional y tiempo estructuralista.

El tejido teórico de estos hilos interpela a Saavedra (2015). El autor señala que la acción interventiva va dirigida en relación a la interpretación de un problema, donde el movimiento que se teje respecto a este, involucra valores, creencias y actitudes, las cuales están situadas en un sistema filosófico y político. Ante esto, ¿Cuál es el problema que se escenifica en la acción? ¿Cuál es el sistema filosófico y político que sustenta dicha acción? Claramente hay un problema declarado, pero pareciera que la interpretación realizada de la acción/ actuación discursada en las orientaciones técnicas va dirigida, entre otras cosas, a controlar las acciones tanto de las intervenidas como de las interventoras. 
Matus (1999) y Carballeda (2008 b), dan cuenta de la característica de control de la intervención social, cuya acción va dirigida a la población a intervenir, mas, estas orientaciones técnicas dan cuenta que la acción de controlar se extiende también a las interventoras. La evaluación coercitiva y el abanico de acción proteccional claramente interpelan a la población declarada como la intervenida, pero hilos dispositivo legal y evidencia panóptica, claramente están interpelando el control hacia la acción/actuación de las interventoras.

Por otro parte, en lo que respecta al sistema filosófico que sustenta y da sentido a la acción/actuación se interpreta que, las orientaciones técnicas, se basan en un paradigma clásico, tal como se mencionó en el apartado anterior. Las orientaciones técnicas están bastante alejadas de una propuesta crítica, pues la actuación/ acción que interpela a las interventoras-intervenidas, apunta a la performance cartesiana-positivista, sujetaobjeto. Esto se interpreta a partir de los hilos discursivos utilitarismo relacional y evaluación coercitiva.

Por otra parte, la actuación de la interventora descrita desde el utilitarismo relacional, permite interpretar una configuración ético-moral de tipo masculino. A partir de los análisis propuestos por Gilligan (1994), la configuración masculina establece que la relación está supeditada a los márgenes normativos declarados en el contexto de interacción, que en este caso corresponde a las orientaciones técnicas. Esto quiere decir que, la relación establecida entre interventora-intervenida/o se encuentra sujeta al fin o al sentido de la creación del programa en la cual basan su interacción, no es una relación que trascienda del contexto que la creó. La relación establecida entre sí, se encuentra con márgenes y cuerpos definidos (Carballeda, 2008 a), pues dicha relación responde exclusivamente al fin por el cual es creado, de ahí que se defina como utilitarismo relacional.

Por último, pareciera que el desarrollo de la perfomance de la intervención está situado y delimitado por ciclo tecnológico de la intervención social y en relación al tiempo.

Butler (1997) define la performance como una interpelación lingüística que crea un cuerpo por medio de un nombre. En lo que respecta al ciclo tecnológico las performance de los nombres están dadas por cada etapa; existe una configuración interventiva diversa según cada una de estas.

Por otra parte, en lo que respecta al tiempo, pareciera ser una categoría con una carga ideológica lo suficientemente intensa como para desnaturalizarla, al punto que no se nombra. Pareciera ser que la idea de tiempo, lineal, es una de las grandes categorías performativas de la intervención, pues da cuenta del inicio, el término, y su presente.

La performance acarrea un poder constitutivo del lenguaje, constituyendo la existencia de la/el sujeto (Butler, 1997), y esta constitución, en este caso, está supeditada al tiempo.

\section{c) Problemas sociales complejos}

Se interpretan los siguientes hilos discursivos a partir de esta categoría: vulneración de derecho, disfuncionalidad parental y comunidad expiada.

Analíticamente el problema social complejo sobre el cual se interviene, posee dos líneas, la declarativa y la no declarativa.

En lo que respecta a la declarativa, las orientaciones técnicas hacen referencia a la vulneración de derechos de NNA, la cual se encuentra definida en relación a la Convención de los derechos del Niño y en relación a decretos de ley. Para Fantova (2007), tal como ya se ha mencionado, la política pública define desde un marco normativo, una necesidad a intervenir, con tal de mejorar la calidad de vida de las personas. A partir de esto, se interpreta que, la necesidad es restituir los derechos vulnerados; el marco normativo que define dicha necesidad, es la Convención de derechos del Niño y las leyes; y, por último, se apunta a mejorar la calidad de vida de los niños, niñas y adolescentes. 
Por otra parte, las orientaciones técnicas apuntan a dos entes responsables de satisfacer las necesidades en relación a los derechos de NNA; estos son la comunidad y la familia. Pero, se ha encontrado en las orientaciones técnicas, a nivel no declarativo que, las acciones y actuaciones interventivas, van dirigidas a un solo elemento definido como problema, la parentalidad y/o negligencia parental.

Se asume entonces que, las acciones de control y disciplina (Carballeda, $2008 \mathrm{~b}$ ) van dirigidas hacia la negligencia parental. Referenciando a Matus (1999), esta disfuncionalidad parental, causante de la vulneración de derecho, debiese estar definida en relación a un marco normativo, el cual debe dar cuenta de la parentalidad ideal, la que no se logra definir en este artículo.

Por su parte, Saavedra (2015), señala que los problemas sociales complejos, se enmarcan dentro de lo que se define como lo deseable y lo no deseable, apelando en el fondo, a lo disfuncional. Por ende, lo disfuncional corresponde a la negligencia parental.

\section{d) Orden Simbólico/ Sistema Normativo}

Los hilos discursivos interpretados bajo esta categoría son: sistema judicial e ideal parental.

La intervención social se construye en relación a un sistema normativo que define el objeto sobre el cual se intervendrá, debido a que escapa de la normativa declarada (Carballeda, 2008 a; Matus, 1999). En este caso, según lo referido por las orientaciones técnicas, el sistema normativo corresponde a lo decretado en la Convención de los derechos del Niño, las leyes que rigen a SENAME y las orientaciones técnicas. Esto permite definir el marco de acción de los programas, puesto que, como refiere Fantova (2007), el criterio normativo permite otorga el marco de acción, de ahí que los telos de los programas estén definidos por decretos de ley.

En el punto anterior se definió como problema social la negligencia parental. Por lo que, bajo la lógica de orden simbólico, faltaría la definición de ideal parental para complementar el sentido de alterización normativa (de Beauvoir, 2011), que, en este caso, corresponde a ideal parental.

\section{e) Actrices/actores de intervención}

Los hilos discursivos interpretados son: interventora como dispositivo material para la intervención, salida de la vulneración y objetualización de la familia.

La configuración de los sujetos de intervención acarrea, nuevamente, el flujo de dinámicas de poder asimétricas, por ende, la intervención descrita en las orientaciones técnicas, se construye a partir de una concepción ontológica de la teoría clásica de la intervención social (Healy, 2001). A esto se le suma la relación alterizada, como plantea García (2003). Este habla de un sujeto cognoscente (interventora e instituciones), y un objeto cognoscible (las/os intervenidas), lo cual permite categorizarlos patológicamente, tal como refiere Matus (1999), desde la significación del sujeto cognoscente.

\section{Análisis hilos discursivos de mujeres interventoras del área de protección de SENAME}

El análisis realizado en este apartado es homologable al análisis recientemente presentado, por lo tanto, los hilos discursivos se construyen a partir de las mismas categorías, pero se realizan sobre los discursos producidos por las mujeres interventoras.

\section{a) Enfoque teórico}

Los hilos discursivos interpretados son: enajenación epistémica, interpelación ética por enajenación epistémica, adscripción axiológica, territorialidad e historicidad como interpelativos teóricos, complejidad epistemológica, hegemonía de la objetividad y sistémica como teoría hegemónica. 
Matus (2006) hace especial énfasis en que la intervención social debe sostenerse dentro de un enfoque epistemológico, puesto que es desde ese enfoque desde el cual se interpreta y comprende la realidad social, así como también marca las directrices de la intervención. Junto con esto, la autora decreta la necesidad que dicho enfoque epistemológico, vaya acompañado de un marco valórico y ético.

Curiosamente, cuatro de las cinco mujeres entrevistadas, dan cuenta de no adscribir a ningún marco epistémico que guíe su práctica. Empero, asumiendo como verdad el párrafo anterior, se interpreta que, en realidad, estas mujeres pudiesen estar enajenadas respecto a su ejercicio. Esta afirmación se sostiene gracias a que a medida que se va tejiendo el discurso, estas dan a conocer una diversidad de posicionamientos epistemológicos y teóricos.

Existe una adherencia a enfoques teóricos de corte modernos, y cartesianos positivistas, que interpelan su ejercicio.

Healy (2001), desde su práctica crítica, refiere la necesidad de visibilizar las relaciones de poder a la base de la interacción, puesto que esto merma y traza el proceso. Al adscribir como interventoras a la práctica clásica/moderna de la intervención social, se desconoce dicho ejercicio de poder, permitiendo asumir, que en la dinámica interventiva, existen sesgos objetualizantes por parte de las interventoras hacia las intervenidas/os.

Por otra parte, al adherir a paradigmas modernos/clásicos de la intervención, se niega la posibilidad crítica reflexiva que acarrea el ejercicio interventivo (Carballeda, 2008 a; Healy, 2001). Dicha adscripción, niega o no agencia reflexión y reconocimiento de un sistema ideológico hegemónico (Healy, 2001), que, al parecer, en el ejercicio de la intervención pareciese ser la teoría de sistemas y la valoración del positivismo.

Complejamente, el enfoque clásico/moderno de la intervención, no es el único que tienen internalizado las mujeres entrevistadas. También llegan a la performatividad de lineamientos de la teoría crítica de la I.S.

Como una práctica no nombrada, las mujeres establecen una relación ética con sus intervenidas/os, llegando a establecer relaciones equitativas, por medio de la identificación, validando la subjetividad del otra/o, pero por, sobre todo, tratando de establecer una práctica e intervención ética y técnica, con tal de contribuir al bienestar del otro. Esto se corresponde a la práctica crítica sugerida por Healy (2001) respecto a la intervención, tales como la promoción de procesos identificatorios, o responsabilizarse ética y técnicamente de la intervención.

La responsabilidad ética para estas mujeres, interpela dos elementos, primero, dominar y profundizar un bagaje teórico, y segundo, generar una relación de cuidado con el otro, por sobre lo que refieren sus lineamientos técnicos.

En lo que respecta cuidado del otro, Gilligan (1994) refiere que este estilo de relacionamiento tiende a configurarse en las mujeres, las cuales, por capacidad empática, ponen en relieve la relación y cuidado del otro por sobre las normas. A esto la autora lo llama moral pragmática.

Por otra parte, en lo que respecta a la necesidad de profundizar en su bagaje teórico técnico, se puede llegar a establecer una relación con la propuesta realizada por Muraro (1994), respecto a la valoración y necesidad de aprehensión hacia los enfoques cientificistas y filosóficos. Esta es una performance que deja en evidencia lo plasmado por la autora, la cual refiere la necesidad de todos de adherir y valorar dicho enfoque como un mandato patriarcal.

\section{b) Actuación/acción de la intervención social.}

Los hilos discursivos interpretados son: actuación según contingencia, escisión teórico-práctica, utilitarismo o ética relacional, tiempo estructuralista y performance del control. 
La mayoría de las entrevistadas da cuenta de una práctica interventiva escindida de la teoría, y con amplia frecuencia de trabajo sobre contingencia.

Si bien Matus (2006) señala que es indispensable reconocer las bases teóricas de la intervención, puesto que guían la práctica, otorgando el marco que sirve para comprenderla y desarrollarla. Pareciese que para algunas de estas mujeres no es necesario del todo, pues su práctica se lleva a cabo de todas formas

Mas, a partir de lo referenciado por Matus (2006), se tiene que, la contingencia y la escisión entre práctica y teoría, pudiese tejer que, a raíz de la ausencia de las consideraciones de la autora, es que se desarrollen esos dos hilos discursivos.

Cabe destacar que una de las entrevistadas otorga total relevancia al posicionamiento teórico, significándolo como la base que da sentido y coherencia a la práctica.

Por otra parte, existe una disociación en lo que respecta a la relación interventiva. Existe una práctica que actúa a la base estratégica presentada por Healy (2001), tales como, procesos de identificación, reconocimiento de dinámicas de poder y valoración por los saberes del otro/a. Mientras que, la acción/actuación hace referencia a un ejercicio clásico, donde prima las relaciones de poder (Healy, 2001), las cuales permiten el control del otro (Carballeda, 2008 b).

Por último, en lo que respecta al tiempo, el análisis es similar, si es que no igual, al realizado en el primer objetivo. En este caso, el tiempo, también interpela la existencia del ejercicio interventivo. Dando lugar a lo que plantea Bulter (1997), donde, el lenguaje -tiempo-, constituye la existencia del sujeto.

\section{c) Problema social complejo de la intervención social}

El hilo discursivo interpretado es la vulneración de derecho.

Tal como se ha mencionado, los problemas sociales con aquellos que se significan como "los/as" no deseables (Saavedra, 2015); en este caso a nivel declarativo, sería la vulneración de derechos a NNA.

Mas, a nivel no declarativo, esto acarrea el surgimiento de otros problemas, la negligencia parental y transgresión de límites de roles familiares.

Ante esto, Matus (2006; 1999), señala que la intervención sobre el problema va dirigida a eliminar la diferencia, con tal de evitar el caos. Se asume, que la negligencia parental y la transgresión de límites familiares, están siendo intervenidos debido a que son significados como caos y desorden para el sistema social. De esto me surge una pregunta, a partir del discurso de las mujeres no se responde ¿Qué clase de rol, como dispositivo, juega la familia dentro de la normativa hegemónica?

Por otra parte, la resistencia de las familias ante la posibilidad de tener que ser normativizadas, activaría, según Matus (2006) un castigo social, caracterizado por la exclusión, o como refiere Fantova (2007), la segregación social. Pero, pareciera que, en realidad, en este caso, el castigo está dirigido hacia la privación afectiva-vincular entre la familia y sus hijos/as; por ende, en este caso no se logra interpretar la exclusión, a salvedad de la afectiva.

\section{d) Orden Simbólico/Sistema Normativo de la intervención Social.}

Los hilos discursivos interpretados son: autoridad interpeladora y agencia subversiva.

Las necesidades interventivas son definidas desde un marco normativo (Fantova, 2007). En este caso, la necesidad definida corresponde a la vulneración de derecho, y el marco normativo, según lo referido por las entrevistadas son, tribunales de familia, representados por magistrados, $y$, por otra parte, las jefaturas técnicas de los programas en los cuales trabajan. 
Según Butler (1997) y Austin (1955), esto se logra debido a que la performance discursiva, se funda por medio de una voz de autoridad, que otorga significado a la realidad. Mas, las mujeres interventoras generan una práctica que escapa de esta voz divina, generando la salida de un sistema normativo relacional y situado, no coaptado por la normativa hegemónica, pero que, como refiere Healy (2001), no puede desconocer la relación de poder entre interventora e intervenida, y norma e interventora.

\section{e) Actrices/actores de intervención}

Los hilos discursivos interpretados bajo esta categoría son: NNA como carentes cautivos, intervención clasista, familia como chivo expiatorio, dinámica relacional v/s dinámica de poder y tribunales de familia como tótem de la intervención.

En relación a tribunales de familia, como sujetos de intervención, se hace una analogía con la teoría freudiana. Freud (1967), realiza estudios antropológicos en los que se dedica a analizar culturas cuyo sistema social es totémico. El autor señala, que el tótem, en estas tribus, simboliza la ley del padre, la autoridad que significa y simboliza el sistema normativo que rige a la tribu y simboliza el dios/animal que los rige. Pero, su normativa y símbolo totémico, no es transmitido por el mismo, sino que por las madres.

La homologación realizada significa a los tribunales como el tótem, que contiene la normatividad de la intervención social, pero que es transmitido por las interventoras.

Por otra parte, en los que respecta a las/os otras/os sujetos de intervención, interventora, NNA o familia, en el análisis, se vislumbra un cierto reduccionismo moderno en lo que respecta a la interpelación que hacen las interventoras de sus intervenidos/as. Esa reducción, primero, apela a la confinación identitaria presente en teorías modernas, descritas por Healy (2001). Y segundo, también responde al reflejo existente entre la interpelación hecha por la interventora hacia la/el intervenida/o (Matus, 2006), donde responde a la categoría asignada por parte de la interventora. Esta categoría estaría restringida a lo que Matus (2006) refiere como patologías sociales "des-afiladas, des-calificadas, de-lincuentes, des-capacitado y des-poseídos".

Por otra parte, la lógica y significación otorgada a la familia por parte de las interventoras, pudiese corresponder a la lógica de la alteridad, donde UNO, representado por las interventoras, objetualiza, exotiza y devalúa al OTRO, (de Beauvoir, 2011). Esto acarrea, inseparablemente, la relación de poder descrita por Healy (2001), entre interventora e intervenida/o.

\section{Conclusiones}

El escenario, declarado, sobre los cuales se sitúan las interventoras, las interpela desde un enfoque clásico de la intervención social (Healy, 2001), lo que acarrea una construcción ontológica objetualizante y patológica respecto a quién se interviene (Matus, 1999). La definición del problema a intervenir se fija desde la normativa institucional (Fantova, 2007), así como también desde la normativa de tribunal de familia. Ambas siguen la lógica de la moral masculinizada (Gilligan, 1994).

Este escenario, declarativo, se entreteje, con las significaciones subjetivas de las interventoras los cuales, si bien, poseen significaciones en torno a la teoría clásica de la intervención social, también generan una performatividad de la teoría crítica de la intervención social (Healy, 2001). A partir de esto, las mujeres interventoras han desplegado una práctica subversiva y de resistencia ante la hegemonía de la intervención, que al parecer no ha sido coaptada, por lo menos lingüísticamente. Esto hace que desplieguen agencia en torno a las estrategias propuestas por Healy (2001), tales como la identificación con el otro, análisis crítico de la práctica, necesidad de una intervención ética-técnica y validación de una moral-ética femenina, según lo descrito por Gilligan (1994). 
La aproximación hacia los procesos de intervención desde el análisis de los discursos de las mujeres interventoras, permite tener una idea de la potencial performativa (Butler, 1997) que se construye en un escenario relevante a nivel de la actualidad nacional, alegórico en lo que respecta a las relaciones de poder, y significativo en la interacción de categorías interseccionales en clase y género.

\section{Bibliografía}

1. Aguilera, E. (2018). Competencias profesionales y buenas prácticas pendientes en trabajadores/as sociales que se desempeñan en contextos de vulneración de derechos de la infancia en la región de Tarapacá. Revista señales, año 11, $n^{\circ} 19,51-65$.

2. Austin, J. (1955). Como hacer cosas con palabras. Santiago de Chile: Escuela de Filosofia Universidad de Arcis.

3. Butler, J. (1997). Lenguaje, poder e identidad. Madrid, España; Sintesis. Y Espacio, 25.

4. Carballeda, A. (2008 a). Problemáticas sociales complejas y políticas públicas. Revista CS, 1, 261-272.

5. Carballeda, A. (2008 b). Los cuerpos fragmentados: la intervención en lo social en los escenarios de la exclusión y el desencanto. Buenos Aires, Argentina: Paidós.

6. Contreras, José Ignacio, Rojas, Vicky, \& Contreras, Lorena. (2015). Análisis de programas relacionados con la intervención en niños, niñas y adolescentes vulnerados en sus derechos: La realidad chilena. Psicoperspectivas, 14(1), 89-102. https://dx.doi.org/10.5027/psicoperspectivas-Vol14-Issue1-fulltext-528

7. De Beauvoir, S. (2011). El segundo sexo. Buenos Aires, Argentina: Siglo XX

8. Egas, V. y Salao, E. (2011). Trabajo comunitario desde una perspectiva psicoanalítica. Un acompañamiento en la construcción grupal de saberes. Revista Latinoamericana de Ciencia Sociales, Niñez y Juventud, 2(9), 899 - 911.

9. Fantova, F. (2007). Repensando la intervención social. Documentación social, 147, 183-198.

10. Freud, S. (1967). Tótem y Tabú.

11. García, C. (2003). La vejez: el grito de los olvidados.D.F. México: Antártica.

12. Gilligan, C. (1994). La moral y la teoría: psicología del desarrollo femenino. D.F., México: Fondo de Cultura Económica.

13. Healy, K. (2001). Trabajo Social: Perspectivas Contemporáneas. Madrid, España: Ediciones Morata.

14. Jäger, S. (2003). Discurso y conocimiento: aspectos teóricos y metodológicos de la crítica del discurso y del análisis de dispositivos. En R. Woodak y M. Meyer (coord.). Métodos de Análisis crítico del discurso. Barcelona, España: Gedisa.

15. Matus, T. (1999): Propuestas contemporáneas en trabajo social: hacia una intervención polifónica. Buenos Aires, Argentina: Espacio editorial.

16. Matus, T. (2006). Apuntes sobre intervención social. Santiago de Chile. Documento Inédito. Extraído de: http://biblioteca.uahurtado.cl/ujah/856/txtcompleto/ txt125117.pdf

17. Molina, V., Moraga, C. (2019). Visitas supervisadas: Una valiosa herramienta de intervención. Revista Señales. $N^{\circ} 20$, vol. XII. $100-117$.

18. Moreno, M. (2013). Psicoanálisis e Intervención Social. Revista CS, 11, 115-142.

19. Muraro, L. (1994). El orden simbólico de la madre. Madrid, España: Cuadernos inacabados.

20. Ortega, M. (2015). Trabajo social como transdisciplina: hacia una teoría de la intervención. Cinta Moebio, 54, 278-289.

21. Saaverdra, J. (2015). Cuatro argumentos sobre el concepto de intervención social Cinta moebio, 53, 135-146.

22. SENAME (2020). Misión y objetivos. Recuperado de: https://www.sename.cl/web/index.php/mision-objetivos/

23. Schöngut-Grollmus, N. (2017). Ensamblajes socio-técnicos para la producción de intervenciones psicosociales en un programa de Servicio Nacional de Menores de Chile. Psicoperspectivas. Individuo y Sociedad, Vol. 16, No. 3.

24. Winnicott, D. (1993). A familia eo desenvolvimiento humano. Sao Paulo, Brasil: Imago.

25. Winnicott, D. (1995). La familia y el desarrollo del individuo. Buenos Aires, Argentina: Horme. 